\title{
Patógenos fúngicos en lesiones dermatológicas de grandes y pequeñas especies animales en clínicas veterinarias y refugios animales en Bogotá D.C.
}

\author{
Fungal pathogens in dermatological lesions of large and small animal species in \\ veterinary clinics and animal shelters in Bogotá D.C. \\ Zully Mariana Peña-Castillo, ${ }^{1,6}$, Adriana Pulido-Villamarínn, ${ }^{1,7}$ Rubiela Castañeda- \\ Salazar ${ }^{1}$, Angélica Barbosa-Buitrago², Beatríz Ortíz ${ }^{3}$, Olimpo Oliver-Espinosa ${ }^{4}$, \\ Myriam Luz Vacca-Sánchez ${ }^{5}$
}

\section{Resumen}

El objetivo del presente estudio fue determinar la presencia de patógenos fúngicos a partir de lesiones dermatológicas en grandes y pequeñas especies animales, en clínicas veterinarias y refugios animales de Bogotá, Colombia. De un total de 30 caninos, 15 felinos y 2 equinos se obtuvieron muestras de raspado de piel (31) e hisopados óticos (26) y de piel (44), que fueron analizadas mediante examen directo, cultivo e identificación a nivel de especie. Se determinó la presencia de Microsporum canis y Malassezia spp. Según la especie animal, predominaron M. canis $(21.4 \%)$ en felinos y Malassezia pachydermatis (32.1\%) en caninos. Según el tipo de muestra, se detectó mayor frecuencia de $M$. canis en raspado de piel (21.4\%) y Malassezia spp en hisopado ótico (21.4\%).

Palabras clave: Malassezia spp, Microsporum canis, felinos, caninos, equinos, dermatitis

\footnotetext{
${ }^{1}$ Semillero en Enfermedades Infecciosas Veterinaria y Zoonosis, Unidad de Investigaciones Agropecuarias -UNIDIA-, Departamento de Microbiología, Facultad de Ciencias, Pontificia Universidad Javeriana, Bogotá, Colombia

${ }^{2}$ Centro Universitario de Atención Veterinaria, Facultad de Medicina Veterinaria, Fundación Universitaria Agraria de Colombia, Bogotá, Colombia

${ }^{3}$ Clínica Veterinaria «de la 53», Bogotá, Colombia

${ }^{4}$ Universidad Nacional de Colombia, Facultad de Medicina Veterinaria y Zootecnia, Departamento de Salud Animal, Bogotá, Colombia

${ }^{5}$ Comevet Pet Spa, Bogotá, Colombia

${ }^{6}$ Email: zullypena@javeriana.edu.co

${ }^{7}$ Email: adriana.pulido@javeriana.edu.co
}

Recibido: 14 de julio de 2020

Aceptado para publicación: 9 de enero de 2021

Publicado: 24 de abril de 2021 
The aim of this study was to determine the presence of fungal pathogens from dermatological lesions of large and small animal species in veterinary clinics and animal shelters in Bogotá, Colombia. From a total of 30 canines, 15 felines and 2 equines, skin scraping samples (31) and otic (26) and skin (44) swabs were obtained, which were analysed by direct examination, culture and identification at the species level. Microsporum canis and Malassezia spp were mainly identified. According to the animal species, M. canis (21.4\%) predominated in felines and Malassezia pachydermatis $(32.1 \%)$ in canines. According to the type of sample, a higher frequency of $M$. canis was detected in skin scraping (21.4\%) and Malassezia spp in ear swabbing $(21.4 \%)$.

Key words: Malassezia spp, Microsporum canis, feline, canine, equine, dermatitis

\section{INTRODUCCIÓN}

Los agentes fúngicos como hongos filamentosos y algunas levaduras han sido considerados como una causa poco probable de infección dermatológica en especies animales; sin embargo, a lo largo del tiempo se han identificado muchos de estos como agentes etiológicos de patologías como la dermatitis, los cuales pueden transmitirse de manera zoonótica afectando al ser humano (Prats, 2012; Galvis y Borda, 2016).

La dermatitis se define como una enfermedad inflamatoria de la piel, originada generalmente por procesos alérgicos, intoxicaciones y por agentes infecciosos que ocasionan una respuesta inflamatoria en la epidermis, evidenciada por prurito, edema, irritación, alopecia e incluso hipo o hiperpigmentación de la zona afectada (Ashbee, 2007; Hossain et al., 2007; Torres et al., 2008). La dermatofitosis es una de las dermatitis causadas por agentes fúngicos, producida por hongos de los géneros Trichophyton, Microsporum y Epidermophyton, más conocidos como dermatofitos, siendo los dos primeros patógenos tanto para animales como humanos (Silva et al., 2003; Frymus et al., 2013; Murmu et al., 2015;
Murray et al., 2015). Adicionalmente, se reportan las dermatomicosis y otomicosis causadas por levaduras del género Malassezia, que, aunque son parte de la microbiota normal de los mamíferos, puede dejar de ser un microorganismo comensal y convertirse en patógeno para el hospedero al presentarse factores predisponentes, como cambios en el microambiente de la piel o en la concentración lipídica (Hernández, 2005; Copetti et al., 2006).

Las dermatofitosis y las dermatomicosis representan las micosis superficiales de mayor importancia en la salud de los animales de compañía y de granja. Se han reportado dermatofitos como $M$. canis y levaduras como Malassezia spp, especialmente $M$. pachydermatis como los principales agentes etiológicos asociados con enfermedades dermatológicas en animales domésticos (Crespo et al., 2002; Copetti et al., 2006; Prado et al, 2008; Rodrígues y Kozusny, 2008). Estas patologías afectan el bienestar animal en todas las especies y generan pérdidas económicas en los sistemas de producción y los animales de granja; además, de representar un riesgo potencial para la salud pública, dada las características zoonóticas de estos microorganismos (Bond, 2010; Moretti et al., 2013). 
En Bogotá, Colombia, se ha determinado que cerca del $20 \%$ de las consultas veterinarias en caninos son debidas a procesos dermatológicos y óticos (Pulido-Villamarín et al., 2010). Las características clínicas de las lesiones en la piel de los caninos se pueden asociar con dermatofitos (86.9\%) y los procesos óticos con Malassezia spp (86.5\%) (Pulido-Villamarín et al., 2011). Por tal motivo, el objetivo de este estudio fue determinar la presencia de patógenos fúngicos en lesiones dermatológicas de grandes y pequeñas especies animales, en instituciones de cuidado y atención veterinaria de Bogotá.

\section{Materiales y Métodos}

Se evaluaron 30 caninos, 15 felinos y 2 equinos, cuyos propietarios consultaron por problemas dermatológicos $\mathrm{u}$ óticos en tres clínicas veterinarias para pequeños animales y en una clínica para grandes animales de la ciudad de Bogotá, o que se encontraban en el Instituto Distrital de Protección y Bienestar Animal (IDPYBA). Según criterio médico, por compatibilidad clínica de las lesiones dermatológicas con sospecha de presencia de agentes fúngicos, se obtuvieron muestras de las lesiones dérmicas.

El muestreo se realizó mediante raspado de piel profundo, colectando escamas y costras de la zona alopécica. Adicionalmente, se obtuvo pelo completo con raíz y se realizaron hisopados en las lesiones exudativas e inflamatorias (Corell, 2006.). En forma similar, se obtuvieron muestras de hisopado ótico. Las muestras de raspado de piel se colectaron en recipientes plásticos estériles y los hisopos fueron introducidos en tubos estériles con Tween 20 al 0.05\% (Pulido-Villamarín et al., 2010). Las muestras fueron transportadas al laboratorio de la Pontificia Universidad Javeriana.

Se realizó análisis directo con hidróxido de potasio - $\mathrm{KOH}$ (Tricograma) a partir de muestras de raspados de piel para determi- nar la presencia de estructuras fúngicas (lesión endotrix y ectotrix) y presencia de ectoparásitos (Curtis, 2001; Pulido-Villamarín et al., 2016). A partir de los hisopados se realizó tinción de Gram para determinar la presencia de levaduras, bacterias y otras estructuras, reportando los resultados mediante la escala semicuantitativa por cruces (PulidoVillamarín et al., 2010). A todas las muestras obtenidas se les hicieron cultivos y aislamientos primarios en agar Dixon, incubando durante 5 días a $32{ }^{\circ} \mathrm{C}$ para la obtención de levaduras lípido-dependientes y agar Sabouraud con cloranfenicol $(\mathrm{Sab}+\mathrm{Clo})$ para determinar la presencia de dermatofitos $u$ otras levaduras, incubando durante máximo 30 días a $25{ }^{\circ} \mathrm{C}$. Una vez obtenidos los aislamientos, se les realizó caracterización macroscópica, microscópica y bioquímica.

En la evaluación de las características macroscópicas de los dermatofitos se tuvo en cuenta el color anverso y reverso de la colonia del hongo, textura, aspecto y presencia de pigmentos difusibles al medio. En las levaduras se consideraron la forma, tamaño, borde, elevación, textura y color de las colonias. La caracterización microscópica se realizó mediante la tinción de Gram y tinción de azul de lactofenol con el fin de evaluar la presencia de estructuras levaduriformes, blastoconidias y/o estructuras fúngicas como hifas y macro-microconidios, respectivamente. Los aislamientos de morfologías compatibles con hongos $(n=27)$ ingresaron al proceso de identificación de especie, utilizando las claves taxonómicas de Barnett y Hunter, 1998 y Guy y Summerbell, 1996.

Para la identificación preliminar de levaduras se realizó la prueba de la ureasa, con la que se determinó si el aislamiento pertenecía al phyllum Basidiomycota o Ascomycota (Batra et al., 2005; Guého-Kellermann et al., 2010). La definición de especie para los aislamientos de Malassezia se realizó a partir de pruebas bioquímicas como catalasa, betaglucosidasa, asimilación de tween, cremophor y crecimiento en Sabouraud; para los aisla- 


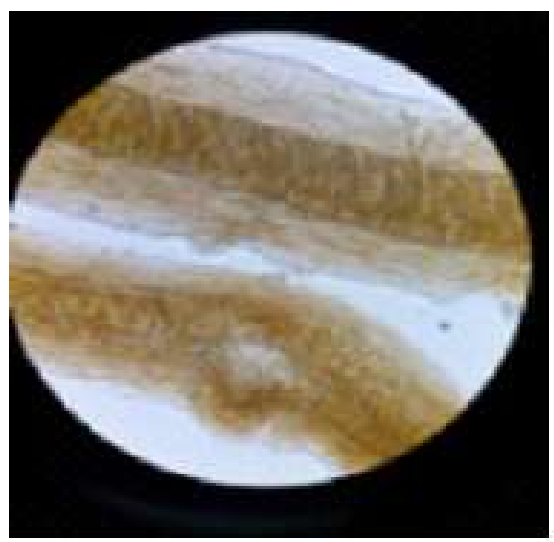

(A)

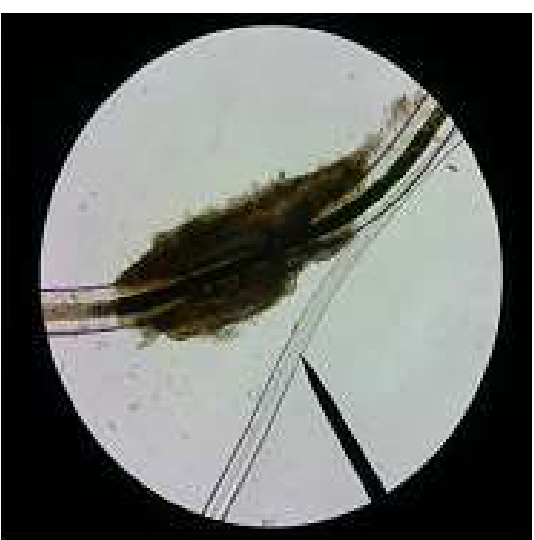

(B)

Figura 1. Visualización de tricograma de lesión en pelo: (A) Lesión endotrix; (B) Lesión ectotrix. 40X

mientos compatibles con Candida spp y otras levaduras se utilizó el medio InTray ${ }^{\mathrm{TM}}$ Colorex ${ }^{\mathrm{TM}}$ Yeast ${ }^{\circledR}$.

\section{Resultados}

De los 47 pacientes con sintomatología dermatológica, se obtuvieron entre 2 a 3 muestras por animal, siendo 44 hisopados de piel, 31 raspados de piel y 26 hisopados óticos, para un total de 101 muestras analizadas. A partir de los tricogramas en las muestras de raspado de piel se evidenció que $16.1 \%(5 / 31)$ presentaban lesión endotrix, 22.6\% (7/31) lesión ectotrix y $29.0 \%$ (9/31) los dos tipos de lesión (Figura 1), mientras que las demás muestras no presentaron lesión en el pelo. Adicionalmente, se evidenció la presencia de ectoparásitos como Demodex spp (1 muestra) y Trichodectes canis (3 muestras).

La presencia de levaduras en hisopados de piel fue en el 11.4\% (5/44) de las muestras y en óticos del $57.7 \%(15 / 26)$, mayormente con dos a tres cruces. Además, se detectó la presencia de bacterias (predominio de cocos Gram positivos) en hisopados óticos $(50.0 \% ; 13 / 26)$ y células epiteliales en hisopados de piel $(4.5 \% ; 2 / 44)$ y ótico $(38.5 \%$; 10/26).

Se obtuvieron 27 aislamientos compatibles con hongos entre filamentosos y levaduriformes. Los hongos filamentosos compatibles con Microsporum canis, presentaron colonias blancas a amarillas y al reverso presentaron color de amarillo a naranja, aspecto radiado y sin pigmento difusible, con macroconidios septados, gruesos, con pared rugosa, ligeramente curvos. Por otro lado, Fusarium spp también es un hongo hialino con macroconidios largos en forma de canoa con presencia de microconidios. El 25.9\% (7/ 27) de los aislamientos se identificó como $M$. canis y el 3.7\% (1/27) como Fusarium spp.

Adicionalmente, se logró identificar 13 aislamientos compatibles con Malassezia spp, con colonias circulares, color beige, borde entero o rizoide, textura cremosa seca y elevadas. La tinción de Gram evidenció levaduras gemantes, que fueron confirmadas mediante la prueba de ureasa positiva, además del panel bioquímico donde se evaluaron los requerimientos para su desarrollo y crecimiento. Se encontró que el $37.0 \%$ (10/27) correspondió a Malassezia pachydermatis, ya que 


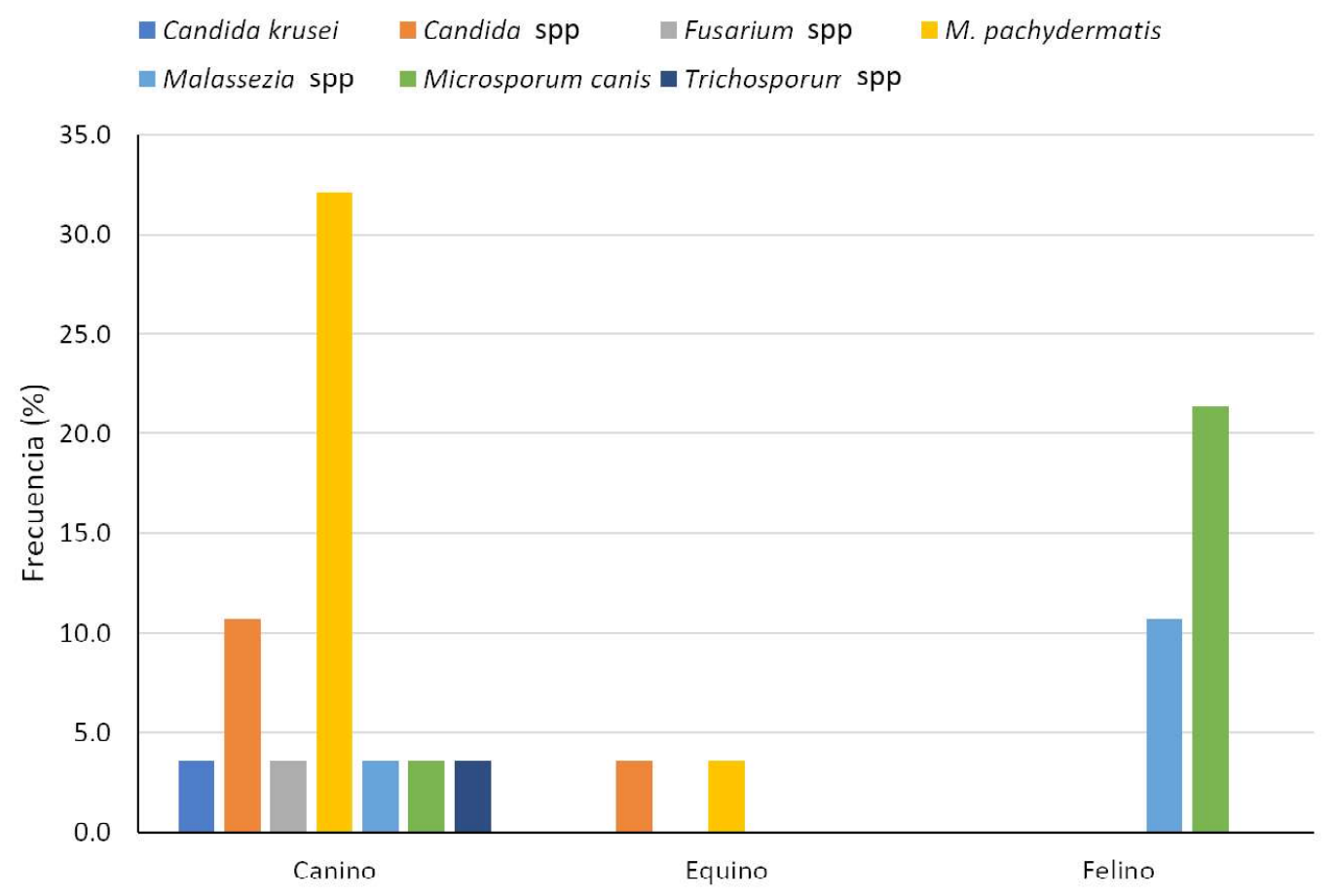

Figura 2. Frecuencia porcentual de agentes fúngicos hallados en lesiones dérmicas de 47 pacientes según especie animal

poseen la capacidad de asimilar todos los tweenes y de crecer en agar Sabouraud con cloranfenicol $(\mathrm{Sab}+\mathrm{Clo})$ a $32{ }^{\circ} \mathrm{C}$, en tanto que $11.1 \%(3 / 27)$ de los aislamientos no presentaron un perfil bioquímico concluyente y fueron designadas como Malassezia spp. De otra parte, seis aislamientos, con morfología macroscópica (colonias con forma circular, color blanco, borde entero, textura cremosa brillante y elevadas) y microscópica compatibles con levaduras, evidenciaron una reacción negativa para ureasa y fueron consideradas como compatibles con Candida spp. Para la identificación a nivel de especie de estos últimos se utilizó el estuche InTray ${ }^{\mathrm{TM}}$ Colorex ${ }^{\mathrm{TM}}$ Yeast, determinando que un aislamiento correspondía a C. krusei, cuatro a Candida spp y uno fue Trichosporon spp.
En el análisis de los aislamientos compatibles con hongos, según la especie animal, partiendo del total de pacientes $(n=47)$ y teniendo en cuenta que se obtuvieron dos tipos de aislamientos a partir de un mismo animal en un canino, un equino y un felino, se determinó la presencia de $M$. canis con predominio en felinos (21.4\%), siendo este el único dermatofito identificado y Malassezia pachydermatis $(32.1 \%)$, principalmente en caninos. En equinos se identificó en un paciente a dos agentes etiológicos: $M$. pachydermatis $(3.6 \%)$ y levaduras del género Candida spp (3.6\%). Además, se aislaron otros microorganismos como Trichosporon spp y Fusarium spp en caninos. De otra parte, no se determinó etiología fúngica asociada con el motivo de consulta en el resto de los pacientes $(\mathrm{n}=22)$ (Figura $2)$. 


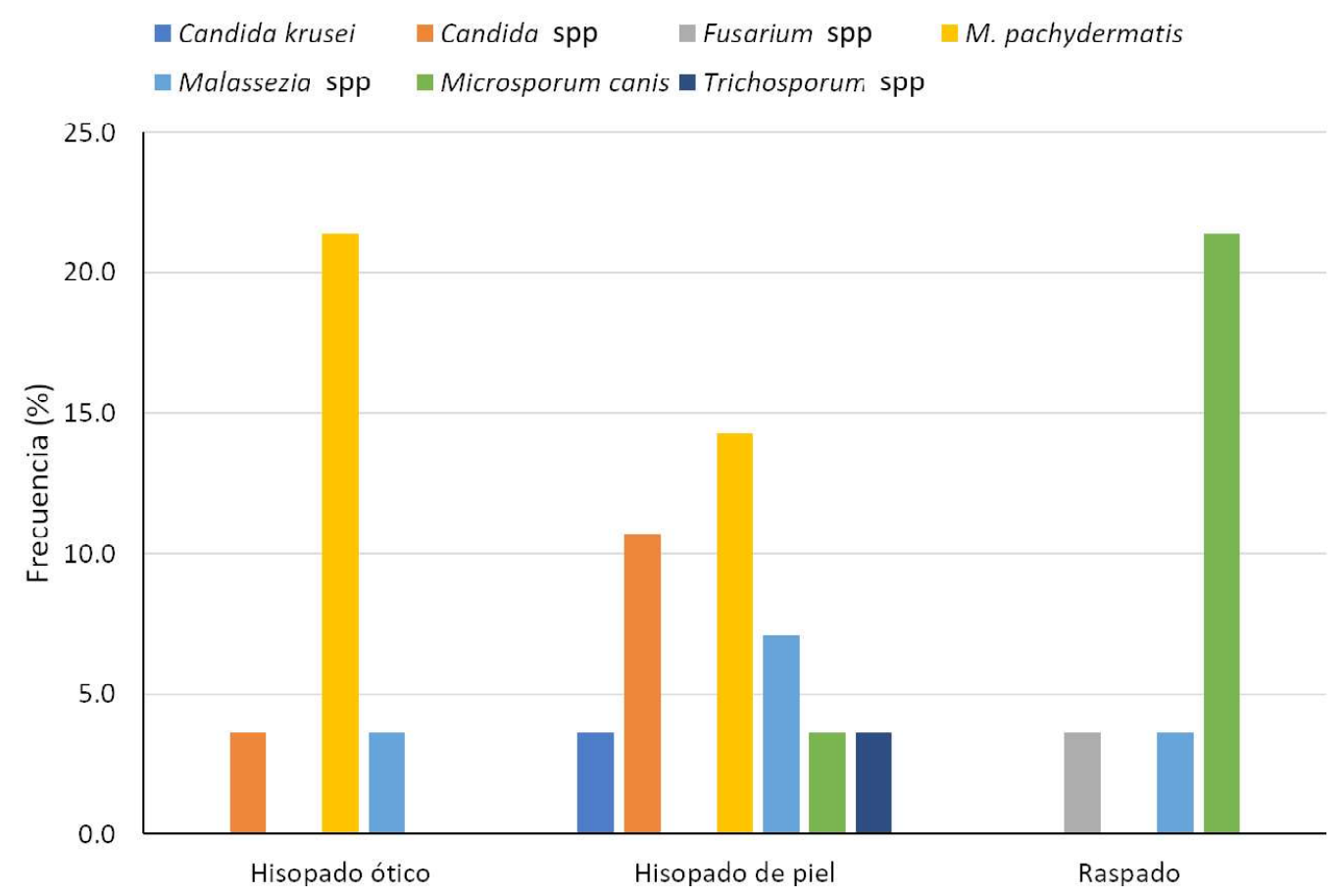

Figura 3. Frecuencia porcentual de hongos hallados en lesiones dérmicas de 47 pacientes según tipo de muestra

En el análisis de los aislamientos de acuerdo con el tipo de muestra se encontró que a partir de 8 hisopados óticos, 12 hisopados de piel y 8 raspados de piel, hubo una mayor frecuencia fúngica en raspado de piel (M. canis [21.4\%]) y de hisopado ótico (Malassezia spp [21.4\%]) (Figura 3).

Únicamente $23.8 \%(5 / 21)$ de 1 los tricogramas positivos con lesión endotrix y ectorix resultaron positivos al cultivo micótico con morfología compatible con M. canis, mientras las 16 muestras restantes no presentaron crecimiento microbiológico. Por otra parte, en las muestras de hisopado (ótico y de piel) se pudo observar una relación entre la positividad citológica y el crecimiento en cultivo, ya que en $17.1 \%$ (12/70) de las citologías positivas para levaduras hubo crecimiento en Agar Dixon, donde se aisló Malassezia spp.

\section{Discusión}

Los microorganismos generalmente implicados como agentes etiológicos en dermatitis infecciosas son hongos dermatofitos y levaduras del género Malassezia (Prado et al., 2008), como se pudo evidenciar en el presente estudio, donde los agentes fúngicos de mayor frecuencia fueron dermatofitos en felinos y Malassezia spp en caninos. López et al. (2012) afirman que $M$. canis puede estar presente en la piel de los animales sin causar lesiones, especialmente en felinos; sin embargo, otros autores indican que su presencia está relacionada con procesos dermatológicos en diversas especies animales (Brilhante et al., 2003; Segundo et al., 2004), tal y como se evidenció en este estudio donde se identificó $M$. canis. Este resultado también concuerda con otros estudios donde se 
identifica con mayor prevalencia en felinos a M. canis (Cabañes, 2000; Moriello, 2014; Murmu et al., 2015; Guerrero et al., 2016).

Malassezia spp (3.67\%) y $M$. pachydermatys $(32.2 \%)$ se identificaron con mayor proporción en caninos con respeto a los felinos (Malassezia spp [10.7\%]), resultado que concuerda con otras investigaciones (Prado et al., 2008; Kagueyama et al., 2016; Luján-Roca et al., 2016).

En equinos, White (2005) reportó que la presencia de Trichophyton equinum, $T$. mentagrophytes, $T$. verrucosum y $M$. equinum en lesiones dérmicas. Asimismo, se reporta especies del género Malassezia (Crespo et al., 2000, Cabañes et al., 2007, Cafarchia et al., 2013; Aldrovandi et al., 2016); sin embargo, en este estudio solamente se determinó la presencia de levaduras $(M$. pachydermatis) en hisopados óticos. Este resultado, no obstante, coincide con otros estudios (White, 2005; White et al., 2006; Castañeda-Salazar et al., 2020) quienes reportan varias especies de Malassezia (M. equi, M. slooffiae, $M$. restricta, $M$. furfur, M. pachydermatis, M. globosa), aunque $M$. pachydermatis es usualmente la más frecuente (Shokri, 2016).

Las levaduras del género Candida aisladas a partir de piel de equino e hisopados óticos y piel de caninos están descritas como microbiota comensal en animales (PulidoVillamarín et al., 2017), incluyendo equinos (Castañeda-Salazar et al., 2020). No obstante, también se ha reportado la presencia de este género de levaduras en caninos con dermatitis, principalmente Candida parapsilosis (Yurayart et al., 2011, 2013; Bumroongthai et al., 2016); sin embargo, en Colombia no se ha reportado la presencia de esta especie como agente involucrado en procesos dermatológicos en animales.

Los resultados obtenidos en este estudio, de acuerdo con el tipo de muestra, evidenciaron que en las lesiones dermatológicas se identificó tanto Malassezia spp como M. canis; mientras a nivel ótico el principal microorganismo identificado fue Malassezia spp; resultados que concuerdan con los reportes de Pulido-Villamarín et al. (2011) y por Copetti et al. (2006). Adicionalmente, en el presente estudio se aisló Fusarium spp, considerado como un hongo ambiental, habitante natural del suelo, patógeno para plantas y humanos (Lainhart, 2018). Sin embargo, se ha reportado su potencial infeccioso en lesiones superficiales en animales (Forero-Reyes et al., 2016).

Los agentes etiológicos identificados en este estudio pueden representar un riesgo de transmisión al ser humano por su carácter zoonótico (Pérez, 2005; Bond, 2010), debido a que no solo se transmiten cuando el animal presenta lesiones, sino que también pueden permanecer en estos hospederos sin generar alteración y pueden ser transmitidos a otros animales y humanos susceptibles de adquirir la infección (Silva et al., 2003; Bond, 2010).

\section{Conclusiones}

- Los principales agentes micóticos asociados con las lesiones dermatológicas en la población estudiada fueron hongos dermatofitos como M. canis y levaduras del género Malassezia.

- Los dermatofitos, principalmente $M$. canis, se aislaron con mayor frecuencia en felinos, mientras las levaduras del género Malassezia spp fueron los agentes predominantes en caninos.

- Los hongos dermatofitos se aislaron con mayor frecuencia en muestras de raspado de piel (21.4\%), mientras Malassezia spp se aisló principalmente de hisopado ótico (21.4\%).

\section{Agradecimientos}

A las clínicas veterinarias que participaron en el estudio y al Instituto Distrital de Protección y Bienestar Animal (IDPYBA), 
a la Dra. Melva Linares por su colaboración, a la Pontificia Universidad Javeriana por la financiación de las actividades del semillero (ID: PTA 00008609) y a la casa comercial BIOTECH por la donación el kit InTray ${ }^{\mathrm{TM}}$ Colorex $^{\mathrm{TM}}$ Yeast ${ }^{\circledR}$.

\section{Literatura Citada}

1. Aldrovandi AL, Osugui L, Coutinho SD. 2016. Is Malassezia nana the main species in horses' ear canal microbiome? Braz J Microbiol 47: 770-774. doi: 10.1016/j.bjm.2016.04.017

2. Ashbee HR. 2007. Update on the genus Malassezia. J Med Microbiol 45: 287303. doi: 10.1080/13693780701191373

3. Barnett HL, Hunter BB. 1998. Illustrated genera of imperfect fungi. St. Paul, Minnesota, USA: The American Phytopathological Society. $218 \mathrm{p}$.

4. Batra R, Boekhout T, Guého E, Cabanes JF, Dawson JR, Gupta AK. 2005. Malassezia Baillon, emerging clinical yeasts. FEMS Yeast Res 5: 11011113. doi: 10.1016/j.femsyr.2005.05.006

5. Bond R. 2010. Superficial veterinary mycoses. Clin Dermatol 28: 226-236. doi: 10.1016/j.clindermatol.2009.12.012

6. Brilhante RS, Cavalcante CS, Soares FA, Cordeiro RA, Sidrim JJ, Rocha MF. 2003. High rate a Microsporum canis feline and canine dermatophytoses in Northeast Brazil: epidemiological and diagnostic features. Mycopathologia 156: 303-308. doi: 10.1023/b:myco.0000003582.67122.69

7. Bumroongthai K, Chetanachan $P$, Niyomtham P, Yurayart C, Prapasarakul N. 2016. Biofilm production and antifungal susceptibility of cocultured Malassezia pachydermatis and Candida parapsilosis isolated from canine seborrheic dermatitis. Sabou-raudia 54: 544-549. doi: 10.1093/mmy/myw002
8. Cabañes FJ. 2000. Dermatofitosis animales. Recientes avances. Rev Iberoam Micol 17: 8-12.

9. Cabañes FJ, Theelsen B, Castellá G, Boektout G. 2007. Two new lipiddependent Malassezia species from domestic animals. FEMS Yeast Res 7: 1064- 1076. doi: 10.1111/j.15671364.2007.00217.x

10. Cafarchia C, Figueredo L, Otranto D. 2013. Fungal diseases of horses. Vet Microbiol 167: 215-234. doi: 10.1016/ j.vetmic.2013.01.015

11. Castañeda-Salazar R, RodríguezSandoval DM, Pulido-Villamarín AP, Linares-Linares MY, Oliver-Espinosa OJ. 2020. Identification of Malassezia species as part of normal skin and ear canal microbiota in horses. Rev Colomb Cienc Pec 33: 15-30. doi: 10.17533/udea.rccp.v33n1a01

12. Copetti MV, Santurio JM, Cavalheiro $A S$, Boeck AS, Argenta JS, Aguiar LC, Alves SH. 2006. Dermatophytes isolated from dogs and cats suspected of dermatophytosis in Southern Brazil. Acta Sci Vet 34: 119-124. doi: 10.22456/ 1679-9216.15173

13. Corell JV. 2006. Micosis cutáneas. Madrid: Médica Panamericana. 200 p.

14. Crespo MJ, Abarca ML, Cabañes FJ. 2002. Occurrence of Malassezia spp in horses and domestic rumiants. Mycoses 45: 333-337. doi: 10.1046/ j.1439-0507.2002.00762.x

15. Curtis CF. 2001. Diagnostic techniques and sample collection. Clin Tech Small An P 16: 199-206. doi: 10.1053/ svms.2001.26998

16. Forero-Reyes CM, AlvaradoFernández AM, Ceballos-Rojas AM, González-Carmona LC, LinaresLinares MY, Castañeda-Salazar R, Pulido-Villamarín AP, et al. 2016. Evaluación de la capacidad patogénica de Fusarium spp en modelos vegetal y murino. Rev Argent Microbiol 50: 90-96. doi: 10.1016/j.ram.2016.11.009 
17. Frymus T, Gruffydd-Jones T, Pennisi MG, Addie D, Belák S, BoucrautBaralon C, Egberink H, et al. 2013. Dermatophytosis in cats: ABCD guidelines on prevention and management. $\mathrm{J}$ Feline Med Surg 15: 598- 604. doi: 10.1177/1098612X13489222

18. Galvis JC, Borda FR. 2016. Infecciones zoonóticas causadas por levaduras del género Malassezia: una revisión. Rev UDCA Actual Div Cient 19: 381 - 393.

19. Guerrero E, González de Cossío A, Segundo C, Cervantes O, Ruiz J, Arenas R. 2016. Microsporum canis y otros dermatofitos aislados de humanos, perros y gatos en la ciudad de México. Glob Dermatol 3: 275-278.

20. Guého-Kellermann E, Boekhout T, Mayser P, Velegraki A. 2010. Malassezia and the skin: science and clinical practice. Berlin: Springer Science \& Business Media. 318 p.

21. Guy St-G, Summerbell R. 1996. Identifying filamentous fungi: a clinical laboratory handbook. Belmont, USA: Star Pub Co. 314 p.

22. Hernández J. 2005. Caracterización molecular de especies del género Malassezia. Tesis doctoral. Barcelona: Univ. Autónoma de Barcelona. $141 \mathrm{p}$.

23. Hossain H, Landgraf V, Weiss R, Mann M, Hayatpor J, Chakraborty T, Mayser P. 2007. Genetic and biochemical characterization of Malassezia pachydermatis with particular attention to pigment- producing subgroups. Med Mycol 45: 41-49. doi: 10.1080/13693780601003827

24. Kagueyama FC, Dias FD, Assunção RJ, Hayakawa IA, da-Silva AD, Cardoso BG, Nakazato, L, et al. 2016. RAPD-based genotyping of Malassezia pachydermatis from domestic and wild animals. Semin-Cienc Agrar 37: 31733180. doi: $10.5433 / 1679-0359.2016 v-$ $37 \mathrm{n} 5 \mathrm{p} 3173$
25. Lainhart, W. 2018. Fusarium spp, a genus of common plant pathogens that can cause devastating, opportunistic human disease. Clin Microbiol Newsl 40: 1-5. doi: 10.1016/j.clinmicnews.2017.12.001

26. López MF, Grilli D, Degarbo S, Arenas $G$, Telechea A. 2012. Frecuencia de dermatofitos en una muestra de felinos del área urbana del Gran Mendoza, Argentina. Rev Iberoam Micol 29: 238-240. doi: 10.1016/j.riam.2012.01.006

27. Luján-Roca DÁ, Saavedra-Espinoza I, Luján-Roca LM. 2016. Frequency of fungi in dogs with mycoses in a veterinary clinic from Callao, Perú. Rev Bio Cien 4: 52-58. doi: 10.15741/ revbio.04.01.05

28. Moretti A, Agnetti F, Mancianti F, Nardoni S, Righi C, Moretta I, Morganti G, Papini M. 2013. Dermatophytoses in animals: epidemiological, clinical and zoonotic aspects. Giorn Ital Dermat V 148: 563-572.

29. Moriello K. 2014. Feline dermatophytosis. J Feline Med Surg 16: 426-431. doi: 10.1177/1098612X14-530215

30. Murmu S, Debnath C, Pramanik K, Mitra T, Jana S, Dey S, Banerjee S, Batabyal K. 2015. Detection and characterization of zoonotic dermatophytes from dogs and cats in and around Kolkata. Vet World 8: 1078-1082. doi: 10.14202/vetworld.2015.1078-1082

31. Murray PR, Rosenthal KS, Pfaller $M A .2015$. Medical microbiology. $8^{\text {th }}$ ed. Philadelphia, USA: Elsevier Health Sciences. $840 \mathrm{p}$.

32. Pérez JC. 2005. Aspectos actuales sobre las dermatofitosis y sus agentes etiológicos. Biosalud 14: 105-121.

33. Prado MR, Brilhante RS, Cordeiro RA, Monteiro AJ, Sidrim JJ, Marcos $M F$. 2008. Frequency of yeasts and dermatophytes from healthy and diseased dogs. J Vet Diagn Invest 20: 197-202. doi: $10.1177 / 104063870802000208$ 
34. Prats G. 2012. Microbiología y parasitología médicas. Madrid, España: Médica Panamericana. $601 \mathrm{p}$.

35. Pulido-Villamarín A, CastañedaSalazar R, Linares-Linares M, Mercado M. 2010. Diagnóstico clínico-microbiológico de otitis externa en caninos de Bogotá-Colombia. Rev MVZ Córdoba 15: 2215-2222.

36. Pulido-Villamarín A, Linares-Linares M, Castañeda-Salazar R, GutiérrezGranados C, Aranda-Silva M, RuedaVarón M. 2011. Análisis retrospectivo (2009-2010) de las alteraciones dermatológicas, óticas y oftalmológicas con diagnóstico clínico presuntivo de micosis en caninos y felinos. Univ Sci 16: 272-281.

37. Pulido-Villamarín A, CastañedaSalazar R, Ibarra-Avila H, GómezMéndez L, Barbosa-Buitrago A. 2016. Microscopía y principales características morfológicas de algunos ectoparásitos de interés veterinario. Rev Inv Vet Perú 27: 91-113. doi: 10.15381/rivep.v27i1.11449

38. Pulido-Villamarín A, DammePedraza S, Castañeda-Salazar R, Linares Linares M, Barbosa-Buitrago A. 2017. Identificación de la microbiota levaduriforme en canal auditivo de porcinos con y sin secreción ótica. Rev Inv Vet Perú 28: 687-695. doi: 10.15381/ rivep.v28i3.13350

39. Rodrígues FD, Kozusny-Andreani, DI. 2008. Isolamento e identificação de fungos associados à dermatofitose $\mathrm{e}$ dermatomicoses em caninos. Ces Med Vet Zootec 3: 58-63.

40. Segundo C, Martínez A, Arenas $R$, Fernández R, Cervantes R. 2004. Dermatomicosis por Microsporum canis en humanos y animales. Rev Iberoam Micol 21: 39-41.
41. Silva V, Thomson P, Maier L, Anticevic S. 2003. Infección y colonización por dermatofitos en cánidos del área sur de Santiago, Chile. Rev Iberoam Micol 20: 145-148.

42. Shokri H. 2016. Occurrence and distribution of Malassezia species on skin and external ear canal of horses. Mycoses 59: 28-33. doi: 10.1111/ myc. 12430

43. Torres E, Arenas R, Atoche C. 2008. Infecciones causadas por el género Malassezia. Med Cutan Iber Lat Am 36: 265-284.

44. White SD. 2005. Equine bacterial and fungal diseases: a diagnostic and therapeutic update. Clin Tech Equine Pract 4: 302-310. doi: 10.1053/j.ctep.2005.10.004

45. White SD, Vandenabeele SI, Drazenovich NL, Foley JE. 2006. Malassezia species isolated from the intermammary and preputial fossa areas of horses. J Vet Intern Med 20: 395-398. doi: 10.1892/0891-6640(2006)20[395:msifti]2.0.co;2

46. Yurayart $C$, Chindamporn $A$, Suradhat S, Tummaruk P, Kajiwara S, Prapasarakul N. 2011. Comparative analysis of the frequency, distribution and population sizes of yeasts associated with canine seborrheic dermatitis and healthy skin. Vet Microbiol 24: 356-362. doi: 10.1016/j.vetmic.2010.09.020

47. Yurayart C, Chindamporn A, Suradhat S, Tummaruk P, Kajiwara S, Prapasarakul N. 2013. Antifungal agent susceptibilities and interpretation of Malassezia pachydermatis and Candida parapsilosis isolated from dogs with and without seborrheic dermatitis skin. J Clin Microbiol 51:721-730. doi: 10.3109/13693786.2013.777165 